\title{
Distributed Queueing Games in Interference-limited Wireless Networks
}

\author{
Zhangyu Guan $^{\dagger \dagger}$, Tommaso Melodia ${ }^{\dagger}$, Gesualdo Scutari ${ }^{\dagger}$ \\ $\dagger^{\dagger}$ Department of Electrical Engineering, \\ State University of New York (SUNY) at Buffalo, Buffalo, NY 14260, USA \\ ${ }^{\ddagger}$ School of Information Science and Engineering, Shandong University, China, 250100 \\ Email:\{zguan2, tmelodia, gesualdo\}@buffalo.edu
}

\begin{abstract}
We study distributed queueing games in interference-limited ad-hoc wireless networks. We formulate system design as a Nash Equilibrium (NE) problem, where the users aim at maximizing their own throughput by choosing the optimal transmission threshold. We first derive conditions for the existence and uniqueness of the $\mathrm{NE}$; then we propose a distributed best-response algorithm solving the game along with its convergence properties.

A second contribution of the paper is to develop a Branch and Bound-based (centralized) algorithm solving the associated (nonconvex) social problem, which one can use as benchmark to evaluate the performance of the proposed game theoretical formulation. Interestingly, our numerical results show that the sum-throughput achievable at the $\mathrm{NE}$ of the proposed game are very close to that of the social problem, which validates our game theoretical formulation. The performance loss is not negligible only in high interference scenarios. For such cases, we proposed a pricing-based algorithm yielding sum-throughput solutions very close to the globally optimal ones, at the cost of very limited signaling among the users.
\end{abstract}

\section{INTRODUCTION}

Two important aspects of the wireless system design is the allocation of the Physical resources and the optimization of the scheduling, based on the queues. Moreover, in infrastructureless wireless networks where there is no centralized control (e.g., mobile ad hoc, vehicular, sensor networks), the above optimization must be performed in a distributed way. When no multiple access scheme is imposed a priori, the users may interfere to each other; which introduces a coupling among the users' transmission strategies. This makes the distributed optimization of the Physical resources and scheduling a challenging problem.

Several game theoretical formulations have been proposed in the literature to deal with the above issue [1]-[8]. For example, in [1], Huang et al. formulated the power control problem in ad hoc networks as a fictitious game; in [2], Park et al. formulated the problem of single-channel carrier sensing as a non-cooperative game; and in [3] SariKaya et al. investigated the problem of uplink wireless random access

This work is based on material supported in part by the National Science Foundation under grants CNS-1055945, CNS-1126357 and CNS-1218717. Zhangyu Guan's work is also supported in part by the NSFC under grant 61101120 and Doctoral Fund of Ministry of Education of China under grant 20110131120028. and formulated it as a dynamic pricing game. However, all these works focus only on the optimization of the physical layer; queuing dynamics have not been incorporated into the analysis. In [4], Le et al. investigated the performance of longest-queue-first (LQF) scheduling for wireless networks under the deterministic SINR interference model; in [5], the authors proposed to maximize the stable throughput of multihop wireless networks with random traffic arrivals based on the back-pressure control policy. However, the SINR interference model in these works primarily captures only the largescale fading characteristics of the signal propagation. In [8], Hanawal et al. formulated stochastic-geometry based medium access games for ad hoc networks as a Nash Equilibrium (NE) problem. The analysis presented in [8] is however restricted to a symmetric game, meaning that all users rely on the same transmission strategy. To the best of our knowledge, the joint and distributed optimization of Physical Layer resources and scheduling based on the queue status is still an open problem (at least under realistic interference model).

This paper is the first attempt toward this direction. We consider a wireless ad-hoc network composed of multiple users sharing the same spectrum. When a new packet arrives, each user decides whether to enqueue the packet, or to transmit it, based on the statistical behavior of the observed interference on each channel. Clearly, the decision of each user will affect also the others. If a node decides to transmit, it generates interference against the other users, thus potentially reducing their packet transmission success rate. On the other hand, if the user enqueues the packet, it increases its queuing delay and therefore the probability of dropping packets (because it violating the maximum delay deadline). A natural question is then how to design distributed algorithms to decide whether to enqueue or transmit that achieve high levels of network throughput?

In this paper we address the above question by focusing on threshold-based policies, where each user selects its "best" instantaneous frequency channel, and then decides to transmit or enqueue by comparing the channel quality with an adaptive threshold; the node transmits if the channel quality is above the threshold, and enqueues otherwise. The reason of using such a transmission strategy is that we are interested in distributed low-complex solution algorithms. The main contributions of 
the paper are the following.

We first formulate the problem of distributed selection of users' transmission thresholds as a non-cooperative game: we model the users as selfish players that compete each other by choosing the optimal transmission threshold maximizing their throughput. We derive sufficient conditions for the existence and uniqueness of a NE, and propose asynchronous bestresponse algorithms converging to the NE.

We then consider a cooperative scenario, in which the users are willing to exchange limited and local signaling information in favor of better performance. In this setting, we formulate the system design as a "social" problem, where the users aim at maximizing the sum-throughput with respect to their transmission thresholds. Since such a formulation is NP hard, we focus on locally optimal solutions, and building on recent results in [9], [10], we propose a novel pricing-based algorithm that converges to a stationary solution of the nonconvex sumthroughput maximization.

Finally we evaluate the performance of the proposed noncooperative and cooperative formulations, using as benchmark the globally optimal solutions achievable by a Branch and Bound-based (centralized) algorithm solving the social problem. The rest of the paper is organized as follows. Section II introduces the system model and problem formulation. In Section III, we present and analyze the proposed best-response distributed algorithm and the pricing-based distributed algorithm. Simulation results are presented in Section IV. Finally, Section V draws some conclusions.

\section{SySTEM MODEL}

We consider a set $\mathcal{N}$ of traffic sessions sharing the same spectrum. For each session, say $n \in \mathcal{N}$, a source-destination pair is identified. Each destination is assumed to be reachable via one-hop by its source node. The spectrum available for communication is divided into a set of $\mathcal{F}$ frequency channels, and the transmission time is divided into consecutive time slots. In each time slot, each backlogged source node can either transmit to its destination by selecting a frequency within $\mathcal{F}$ or be silent and enqueue any new packets in its buffer.

We consider a threshold-based transmission policy, according to which each user (i) selects the frequency channel with the best channel gain, say $f^{*}$, and (ii) transmits a packet to its destination over $f^{*}$ if the corresponding channel gain is higher than a threshold $\beta$, and does not transmit otherwise. Note that each channel is potentially shared by different sessions (thus interfering to each other). A higher threshold $\beta$ implies higher probability of good channel quality and hence lower rate of transmission error. However, a higher threshold also reduces opportunities for a node to transmit. This may result in a larger queueing delay, and consequently in a higher probability that a packet exceed the maximum queueing delay and then be dropped at the receiver side. Conversely, a lower threshold $\beta$ implies higher transmission error rate, but lower packet dropping rate. Therefore, each user needs to dynamically adjust its channel gain threshold $\beta$ to explore the optimal tradeoff between transmission and queueing.
Channel model. Denoting by $h_{n}^{f}$ the channel gain on frequency $f \in \mathcal{F}$ of user $n \in \mathcal{N}$ between its source and destination nodes, $h_{n}^{f}$ can be written as $h_{n}^{f}=\hat{h}_{n} \tilde{h}_{n}^{f}$, where $\hat{h}_{n}$ represents the square root of path loss and $\tilde{h}_{n}^{f}$ is the channel fading coefficient. Considering a non-singular path loss $\operatorname{model}^{1}$, then $\hat{h}_{n}=\sqrt{\left(1+l_{n}^{\alpha}\right)^{-1}}$, where $l_{n}$ is the communication distance $[\mathrm{m}]$ between the transmitter and the receiver of user $n$, and $\alpha$ represents the path loss factor. We assume a block fading channel, i.e., the channel fading coefficients $\tilde{h}_{n m}^{f}$ change at the beginning of each time slot and remain constant for the entire duration of a time slot. In each time slot, each $\tilde{h}_{n m}^{f}$ is assumed to be Rayleigh distributed with parameter $\Omega$, i.e.,

$$
\operatorname{Pb}\left(\tilde{h}_{n m}^{f}=x\right)=\frac{2 x}{\Omega} e^{-\frac{x^{2}}{\Omega}}, f \in \mathcal{F}, \forall n, m \in \mathcal{N} .
$$

Threshold policy. Let $\beta_{n}>0$ be the channel fading threshold for user $n \in \mathcal{N}$ and let $f^{*}$ be the best frequency channel, i.e., $f^{*}=\arg \max _{f \in \mathcal{F}} \hat{h}_{n} \tilde{h}_{n}^{f}$. Then, according to the threshold policy, user $n$ chooses to transmit a packet over channel $f^{*}$ if $\tilde{h}_{n}^{f^{*}} \geq \beta_{n}$ and to queue otherwise. Then, according to (1), the probability that $\tilde{h}_{n}^{f}$ is lower than the threshold $\beta_{n}$ can be expressed as

$$
\operatorname{Pb}\left(\tilde{h}_{n}^{f}<\beta_{n}\right)=\int_{0}^{\beta_{n}} \frac{2 x}{\Omega} e^{-\frac{x^{2}}{\Omega}} d x=1-e^{-\frac{\beta_{n}^{2}}{\Omega}} .
$$

The probability that user $n \in \mathcal{N}$ transmits a packet during a time slot, denoted as $\phi\left(\beta_{n}\right)=1-\operatorname{Pb}\left(\tilde{h}_{n}^{f^{*}}<\beta_{n}\right)$, is then

$$
\phi\left(\beta_{n}\right)=1-\left(1-e^{-\frac{\beta_{n}^{2}}{\Omega}}\right)^{|\mathcal{F}|}
$$

where $|\mathcal{F}|$ represents the cardinality of the set $\mathcal{F}$.

Queuing model. Let $\nu_{n}$ denote the number of time slots that it takes for node $n \in \mathcal{N}$ to transmit a packet. The probability density function (PDF) of $\nu_{n}$ is

$$
\operatorname{Pb}\left(\nu_{n}=k\right)=\left(1-\phi\left(\beta_{n}\right)\right)^{k-1} \phi\left(\beta_{n}\right) .
$$

The expected value of $\nu_{n}$, denoted by $\mathrm{E}\left[\nu_{n}\right]$, is $\mathrm{E}\left[\nu_{n}\right]=\frac{1}{\phi\left(\beta_{n}\right)}$.

To keep our analysis tractable, we approximate the pdf in (4) using an exponential distribution having the same firstorder moment of the original pdf. Denoting by $\mu_{n}\left(\beta_{n}\right)$ the parameter of the exponential distribution function associated with user $n \in \mathcal{N}$, the pdf approximating (4) is

$$
\operatorname{Pb}\left(\nu_{n}=k\right) \approx \mu_{n}\left(\beta_{n}\right) e^{-\mu_{n}\left(\beta_{n}\right) k},
$$

where $\mu_{n}\left(\beta_{n}\right)=\phi\left(\beta_{n}\right)$ with $\phi\left(\beta_{n}\right)$ defined in (3). Numerical results show that (5) fits very well the original pdf in (4) [12].

Assuming that the packet arrival rate at each node $n \in \mathcal{N}$ follows a Poisson distribution with average rate $\lambda_{n}$, the queue at node $n \in \mathcal{N}$ can be modeled as an $M / M / 1$ queue, based on the approximation in (5). Denoting by $T_{n}^{\text {th }}$ the maximum queuing delay for user $n \in \mathcal{N}$, the packet loss probability

\footnotetext{
${ }^{1}$ In the non-singular path loss model, the path loss tends to one as propagation distance tends to zero, whereas in singular path model, the path loss tends to infinity. Readers are referred to [11] for details.
} 
of user $n$ caused by exceeding the maximum queueing delay $T_{n}^{\mathrm{th}}$, denoted by $P_{d l y}^{n}\left(\beta_{n}\right)$, is

$$
P_{d l y}^{n}\left(\beta_{n}\right) \triangleq \operatorname{Pb}\left(T_{n}>T_{n}^{\mathrm{th}}\right)=e^{-\left(\frac{\mu_{n}\left(\beta_{n}\right)}{T_{s l t}}-\lambda_{n}\right) T_{n}^{\mathrm{th}}},
$$

where $T_{s l t}$ is the time duration of a time slot. Since $P_{d l y}^{n}\left(\beta_{n}\right)$ in (6) can not be greater than 1 , it must be

$$
\frac{\mu_{n}\left(\beta_{n}\right)}{T_{s l t}}-\lambda_{n} \geq 0,
$$

with $\mu_{n}\left(\beta_{n}\right)=\phi\left(\beta_{n}\right)$ defined in (3). This leads to the following upper bound on $\beta_{n}$

$$
\beta_{n} \leq \beta_{n}^{\mathrm{U}}, \forall n \in \mathcal{N},
$$

with

$$
\beta_{n}^{\mathrm{U}}=\sqrt{-\ln \left[1-\left(1-T_{\text {slt }} \lambda_{n}\right)^{\frac{1}{|\mathcal{F}|}}\right] \Omega} .
$$

Interference model. If multiple users select the same frequency channel, they will interfer to each other. Let $\gamma_{\text {th }}$ be the threshold on the signal-to-noise-plus-interference ratio (SINR) $\gamma_{n}$ above which a packet can be correctly decoded at a receiver, Then, the probability that a transmission error occurs for user $n \in \mathcal{N}$ is

$$
\operatorname{Pb}\left(\gamma_{n}<\gamma_{\text {th }}\right)=\operatorname{Pb}\left(\frac{P_{n} \hat{h}_{n}^{2}\left(\tilde{h}_{n}^{f}\right)^{2}}{\sigma_{n, f}^{2}+I_{n}^{f}\left(\boldsymbol{\beta}_{-n}\right)}<\gamma_{\text {th }}\right),
$$

where $P_{n}$ is the transmission power of user $n \in \mathcal{N}, \sigma_{n, f}^{2}$ is the power of Gaussian noise, and $I_{n}^{f}\left(\boldsymbol{\beta}_{-n}\right)$ represents the interference at the destination node of user $n$ on the carrier $f \in \mathcal{F}$, which depends on the transmission thresholds $\boldsymbol{\beta}_{-n} \triangleq$ $\left(\beta_{m}\right)_{m \in \mathcal{N} / n}$. In (10), the channel fading $\tilde{h}_{n}^{f}$ follows a Rayleigh distribution as in (1) and takes values in $\left[\beta_{n}+\infty\right)$, where $\beta_{n}$ is the transmission threshold of user $n$.

The interference $I_{n}^{f}\left(\boldsymbol{\beta}_{-n}\right)$ in (10) can be expressed as

$$
I_{n}^{f}\left(\boldsymbol{\beta}_{-n}\right)=\sum_{m \in \mathcal{N} / n} P_{m} \hat{h}_{m n}^{2}\left(\tilde{h}_{m n}^{f}\right)^{2} \alpha_{m}^{f}\left(\beta_{m}\right),
$$

where $\hat{h}_{m n}^{2}$ and $\left(\tilde{h}_{m n}^{f}\right)^{2}$ represent the path loss and the square of channel fading between the source node $m \in \mathcal{N}$ and the destination node $n \in \mathcal{N}$; the value of $\alpha_{m}^{f}\left(\beta_{m}\right)$ is equal to one if user $m$ transmits, and zero otherwise. Therefore, the aggregate interference measured at the receiver node of user $n$ depends on (i) the locations of the source nodes of all other sessions, (ii) whether each source transmits or not (iii) which channel each source uses for transmission.

We model the distribution of $I_{n}^{f}\left(\boldsymbol{\beta}_{-n}\right)$ in (11) following a classical stochastic geometry theory approach [13]. More specifically, the node distribution in the network are assumed to follow a bi-dimensional Poisson Point Process (PPP). This is a well-accepted model of static ad-hoc networks with random deployment as well as networks with moving users. In this setting, the pdf of $I_{n}^{f}\left(\boldsymbol{\beta}_{-n}\right)$ is the Gamma distribution function $\zeta_{n}(x)$, i.e.,

$$
\begin{aligned}
\zeta_{n}(x) & \triangleq \operatorname{Pb}\left(I_{n}^{f}\left(\boldsymbol{\beta}_{-n}\right)=x\right) \\
& =x^{k_{n}\left(\boldsymbol{\beta}_{-n}\right)-1} \frac{e^{-x / \theta_{n}\left(\boldsymbol{\beta}_{-n}\right)}}{\Gamma\left(k_{n}\left(\boldsymbol{\beta}_{-n}\right)\right) \theta_{n}^{k_{n}\left(\boldsymbol{\beta}_{-n}\right)}\left(\boldsymbol{\beta}_{-n}\right)},
\end{aligned}
$$

where $k_{n}\left(\boldsymbol{\beta}_{-n}\right)$ and $\theta_{n}\left(\boldsymbol{\beta}_{-n}\right)$ are $\boldsymbol{\beta}_{-n}$-dependent shaping parameters of the Gamma function, and both can be estimated online; and $\Gamma\left(k_{n}\right)$ in (12) is given by

$$
\Gamma\left(k_{n}\left(\boldsymbol{\beta}_{-n}\right)\right)=\int_{0}^{\infty} x^{k_{n}\left(\boldsymbol{\beta}_{-n}\right)-1} e^{-x} d x .
$$

Let $\vartheta_{n}\left(x, \boldsymbol{\beta}_{-n}\right)$ be the complementary cumulative distribution function of $I_{n}^{f}\left(\boldsymbol{\beta}_{-n}\right)$. Then, according to (12), $\vartheta_{n}\left(x, \boldsymbol{\beta}_{-n}\right)$ can be written as

$$
\begin{aligned}
\vartheta_{n}\left(x, \boldsymbol{\beta}_{-n}\right) & \triangleq \operatorname{Pb}\left(I_{n}^{f}\left(\boldsymbol{\beta}_{-n}\right)>x\right) \\
& =1-\frac{\varphi\left(k_{n}\left(\boldsymbol{\beta}_{-n}\right), \frac{x}{\theta_{n}\left(\boldsymbol{\beta}_{-n}\right)}\right)}{\Gamma\left(k_{n}\left(\boldsymbol{\beta}_{-n}\right)\right)},
\end{aligned}
$$

where $\Gamma\left(k_{n}\left(\boldsymbol{\beta}_{-n}\right)\right)$ is defined in (13), and $\varphi\left(k_{n}\left(\boldsymbol{\beta}_{-n}\right), \frac{x}{\theta_{n}\left(\boldsymbol{\beta}_{-n}\right)}\right)$ is the incomplete gamma function given by

$$
\varphi\left(k_{n}\left(\boldsymbol{\beta}_{-n}\right), \frac{x}{\theta_{n}\left(\boldsymbol{\beta}_{-n}\right)}\right)=\int_{0}^{\frac{x}{\theta_{n}\left(\boldsymbol{\beta}_{-n}\right)}} s^{k_{n}\left(\boldsymbol{\beta}_{-n}\right)-1} e^{-s} d s .
$$

Under the above assumptions, the packet loss probability in (10) is given by

$$
\begin{aligned}
& P_{\text {err }}^{n}(\boldsymbol{\beta}) \triangleq \operatorname{Pb}\left(\gamma_{n}<\gamma_{\mathrm{th}}\right) \\
= & \int_{\beta_{n}}^{\infty} \frac{2 x}{\Omega} e^{-\frac{x^{2}}{\Omega}} \vartheta_{n}\left(\frac{P_{n} \hat{h}_{n}^{2}}{\gamma_{\mathrm{th}}} x^{2}-\sigma_{n, f}^{2}, \boldsymbol{\beta}_{-n}\right) d x
\end{aligned}
$$

with $\vartheta_{n}(\cdot, \cdot)$ defined in (14) and $\boldsymbol{\beta} \triangleq\left(\beta_{n}\right)_{n \in \mathcal{N}}$.

Expected throughput. Using the expressions of the packet loss probability $P_{d l y}^{n}\left(\beta_{n}\right)$ and the transmission error rate $P_{e r r}^{n}(\boldsymbol{\beta})$ of user $n$ as given in (6) and (16), respectively, we can now introduce the overall loss rate $P_{\text {los }}^{n}(\boldsymbol{\beta})$ and the expected throughput $R_{n}(\boldsymbol{\beta})$ of each user $n$, given by

$$
\begin{aligned}
& P_{l o s}^{n}(\boldsymbol{\beta})=P_{d l y}^{n}\left(\beta_{n}\right)+\left[1-P_{d l y}^{n}\left(\beta_{n}\right)\right] P_{e r r}^{n}(\boldsymbol{\beta}) \\
& R_{n}(\boldsymbol{\beta})=\lambda_{n}\left(1-P_{d l y}^{n}\left(\beta_{n}\right)-\left[1-P_{d l y}^{n}\left(\beta_{n}\right)\right] P_{e r r}^{n}(\boldsymbol{\beta})\right),
\end{aligned}
$$

where $\lambda_{n}$ is the average incoming packet rate of user $n$. Note that $R_{n}(\boldsymbol{\beta})$ is a function of $\boldsymbol{\beta}$ through $P_{d l y}^{n}\left(\beta_{n}\right)$ and $P_{e r r}^{n}(\boldsymbol{\beta})$.

We now approximate the throughput $R_{n}(\boldsymbol{\beta})$ in (18) by neglecting the second-order term $P_{d l y}^{n}\left(\beta_{n}\right) P_{e r r}^{n}(\boldsymbol{\beta})$, which is acceptable when the overall packet loss rate is low or moderate. For example, if $P_{d l y}^{n}\left(\beta_{n}\right)=0.1$ and $P_{e r r}^{n}(\boldsymbol{\beta})=0.1$ (which are already very high values), the overall packet loss rate is 0.19 and the approximation is 0.20 . The resulting approximation error is only 0.01 . The approximation error is 0.1 when $P_{d l y}^{n}\left(\beta_{n}\right)$ and $P_{e r r}^{n}(\boldsymbol{\beta})$ take a value of 0.2 . Based on the approximation, the throughput of user $n \in \mathcal{N}$ can be simplified as

$$
R_{n}(\boldsymbol{\beta})=\lambda_{n}\left(1-P_{d l y}^{n}\left(\beta_{n}\right)-P_{\text {err }}^{n}(\boldsymbol{\beta})\right) .
$$

System design. The system design would be to maximize the sum throughput of all users in $\mathcal{N}$, i.e.,

$$
\begin{array}{cl}
\max _{\boldsymbol{\beta}=\left(\beta_{n}\right)_{n \in \mathcal{N}}} & \sum_{n \in \mathcal{N}} R_{n}(\boldsymbol{\beta}) \\
\text { s. t. } & \beta_{n} \geq 0, \forall n \in \mathcal{N} \\
& \beta_{n} \leq \beta_{n}^{\mathrm{U}}, \forall n \in \mathcal{N}
\end{array}
$$


However, the above optimization problem is highly nonconvex, implying that only suboptimal solutions can be efficiently computed even with centralized algorithms. Since we would like to design distributed solutions with low complexity, we follow here a different approach and we concentrate on decentralized strategies where the users are able to selfenforce the negotiated agreements on the use of the available spectrum without the intervention of a centralized authority. This motivates and makes natural a game-theoretic approach to this multi-agent decision making problem.

\section{DistRibuted System Design}

In this section, we first formulate the system design as a Nash equilibrium problem and propose a distributed algorithm to compute the NE of the game. Then we focus on a cooperative scenario where the users are willing to exchange some informations in favor of better performance, and design a pricing-based algorithm converging to a stationary solution of (20).

\section{A. Game Theoretical Formulation}

We formulate the optimization of the channel thresholds as a NE Problem, where the users represent the players of the game and the payoff function of each user is the achievable throughput $R_{n}(\boldsymbol{\beta})$; each player $n \in \mathcal{N}$ competes against the others by choosing the channel threshold $\beta_{n}$ that maximizes its own throughput $R_{n}(\boldsymbol{\beta})$, i.e.,

$$
\begin{array}{cl}
\max _{\beta_{n} \in \Phi_{n}} & R_{n}\left(\beta_{n}, \boldsymbol{\beta}_{-n}\right) \\
\text { s.t. } & \beta_{n} \geq \beta_{n}^{\mathrm{L}} \\
& \beta_{n} \leq \beta_{n}^{\mathrm{U}}
\end{array}
$$

where $\beta_{n}^{\mathrm{U}}$ is the upper bound of $\beta_{n}$ as given in (9), $\beta_{n}^{\mathrm{L}}$ is a given lower bound (we discuss shortly how to choose this lower bound), and $\Phi=\prod_{n \in \mathcal{N}} \Phi_{n}$ with $\Phi_{n}=\left[\begin{array}{ll}\beta_{n}^{\mathrm{L}} & \beta_{n}^{\mathrm{U}}\end{array}\right]$ denotes the joint strategy set of the game.

Definition 1 (Nash Equilibrium): A channel fading threshold vector $\boldsymbol{\beta}^{*}=\left(\beta_{n}^{*}\right)_{n \in \mathcal{N}}$ is a NE of the game (21) if the following condition holds for all the users $n \in \mathcal{N}$ :

$$
R_{n}\left(\beta_{n}^{\star}, \boldsymbol{\beta}_{-n}^{\star}\right) \geq R_{n}\left(\beta_{n}, \boldsymbol{\beta}_{-n}^{\star}\right), \forall \beta_{n} \in \Phi_{n} .
$$

Theorem 1 (Existence of $N E$ ): Given the NE Problem (21), suppose that $\beta_{n}^{\mathrm{L}} \geq \frac{\sqrt{2}}{2} \Omega$ for all $n \in \mathcal{N}$, where $\Omega$ is the Rayleigh fading factor. Then, the NE Problem has a NE.

Proof: The existence of a NE is guaranteed if i) the strategy set of each player is convex and compact; and ii) the payoff function of each player is a continuous function of $\boldsymbol{\beta}$ and concave in $\beta_{n}$, for any given feasible $\boldsymbol{\beta}_{-n}$ [14]. We only need to show that each function $R\left(\beta_{n}, \boldsymbol{\beta}_{-n}\right)$ is a concave function of $\left[\beta_{n}^{\mathrm{L}}, \beta_{n}^{\mathrm{U}}\right]$, when $\beta_{n}^{\mathrm{U}} \geq \frac{\sqrt{2}}{2} \Omega$. This can be easily checked computing the first and second derivatives of $P_{l o s}^{n}(\boldsymbol{\beta})$ with respect to $\beta_{n}$ which is omitted because of the space limitation.

Distributed best-response algorithms. To compute a NE we focus on Jacobi best-response algorithms: all the users simultaneously update their strategies solving in parallel their own (convex) optimization problems (21). The formal description of the algorithm is given in Algorithm 1.

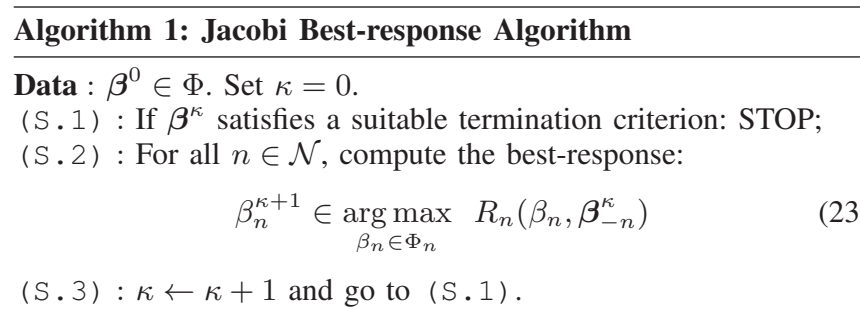

Convergence conditions of Algorithm 1 are given in Theorem 2 below, whose proof is based on recent results in [15] (see also [16] for more details) and is omitted because of the space limitation.

Theorem 2 (Convergence Condition): Given the game in (21) in the setting of Theorem 1, suppose that the following conditions are satisfied:

$$
\min _{\boldsymbol{\beta} \in \Phi} \frac{\partial^{2} P_{l o s}^{n}(\boldsymbol{\beta})}{\partial \beta_{n}^{2}}>\sum_{m \in \mathcal{N} / n} \max _{\boldsymbol{\beta} \in \Phi} \frac{\partial^{2} P_{l o s}^{m}(\boldsymbol{\beta})}{\partial \beta_{m} \partial \beta_{n}}, \forall n \in \mathcal{N} .
$$

Then, the sequence generated by Algorithm 1 converges to the unique NE of the game.

Note that the convergence conditions in Theorem 2 do not depend on the specific updating order performed by the users. In fact, we can prove that these conditions guarantee also convergence of best-response algorithms based on a totally asynchronous update of the player strategies (in the sense of [16]). In such asynchronous schemes, some players may change their strategies more frequently than the others (e.g., at random times) and they may even use an outdated information of the other players strategies, without affecting the convergence of the algorithm. It turns out that instances of the aforementioned asynchronous framework are robust against missing or outdated updates of the players. This feature strongly relaxes the constraints on the network synchronization; which makes the proposed class of algorithms truly appealing in many practical scenarios. Interestingly the convergence conditions in (24) have an intuitive interpretation; they define different network scenarios wherein the algorithm is guaranteed to converge, as detailed next.

Congestion-dominated wireless network. This scenario corresponds to large incoming average packet rates $\lambda_{n}$. Indeed, the analysis of (24) shows that the left-hand side of the inequality monotonically increases with $\lambda_{n}$, implying that condition (24) tends to be satisfied in networks with large $\lambda_{n}$, where the users tend to choose low transmission thresholds $\beta_{n}$ (they transmit more often to avoid high packet loss rate due to exceeding the the maximum queueing delay $T_{n}^{\text {th }}$ ).

Loosely coupled interference. This corresponds to sparse wireless networks (e.g., due to low density of nodes or large number of available channels). When this happens, the righthand side of (24) tends to be "very small", making the convergence condition more likely to be satisfied.

Delay-insensitive wireless networks. In this setting, we have large maximum tolerable queueing delays $T_{n}^{\text {th }}$. Indeed, 


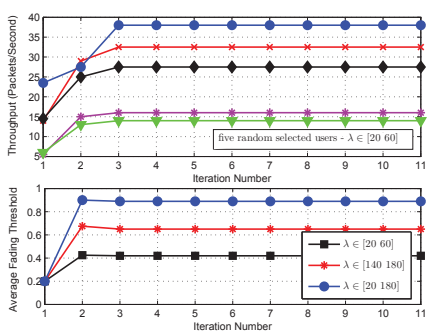

(a)

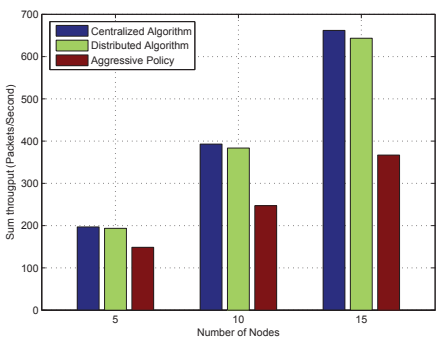

(b)

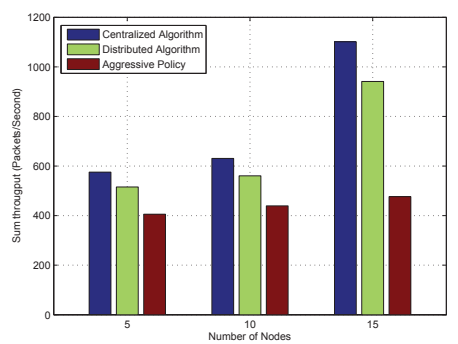

(c)

Fig. 1: (a): Convergence of the best-response based distributed algorithm (top) and average rayleigh fading threshold (bottom); (b) Performance of the three algorithms in the case of low traffic load and ten frequencies. (c) Performance comparison in the case of moderate load and two frequencies.

when $T_{n}^{\text {th }}$ s are "large", the left-hand side of (24) tends to be "large" too, making the convergence condition to be satisfied. In this scenario, each user will choose to enqueue its data more often and transmit only when its channel quality is very good, resulting in low generated interference.

\section{B. Pricing-based Algorithms}

We assume now that the users are willing to exchange some limited signaling in favor of better performance. Building on recent results in [9], [10], we proposed next a bestresponse Jacobi algorithm based on pricing that converges to a stationary solution of the social problem (20). The main idea of the algorithm is to implement in a distributed way a pricing mechanism; each user $n$ maximizes his own rate minus a pricing term that measures somehow the marginal increase of the sum-utility of the other users due to a variation of the strategy of user $n$. Roughly speaking, the pricing works like a punishment imposed to each user for being too aggressive in choosing his own strategy and thus "hurting" the other users. The formal description of the algorithm is given in Algorithm 2 , where the pricing term $\pi_{n}\left(\boldsymbol{\beta}^{\nu}\right)$ in (26) is defined as

$$
\pi_{n}\left(\boldsymbol{\beta}^{\kappa}\right)=\sum_{m \in \mathcal{N} / n} \omega_{m} \nabla_{\beta_{n}} R_{m}\left(\boldsymbol{\beta}^{\kappa}\right),
$$

and $\frac{\tau_{n}}{2}\left(\beta_{n}-\beta_{n}^{\kappa}\right)^{2}$, with $\tau>0$, is a proximal regularization term, whose numerical benefits are well-known [17].

The convergence properties of Algorithm 2 are given in Theorem 3 below. The proof of the theorem is based on [9], [10] and is omitted because of space limitation.

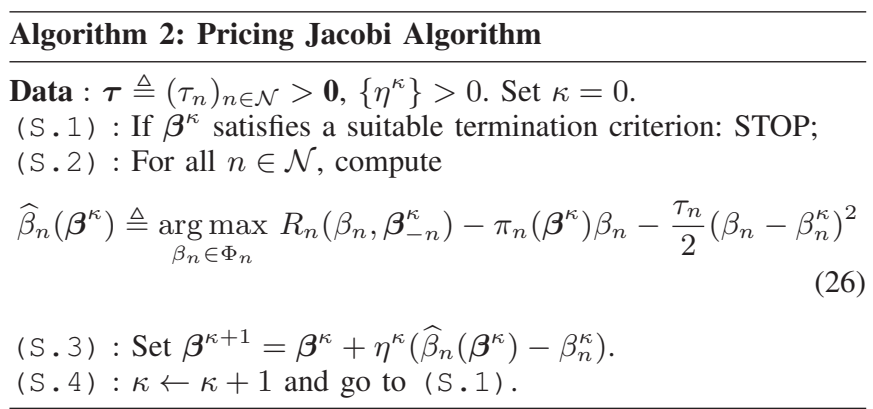

Theorem 3 (Convergence Condition): Given the social problem (20), suppose that $\left\{\eta_{\kappa}\right\}$ is choose so that

$$
\eta^{\kappa} \in(0,1], \eta^{\kappa} \rightarrow 0, \text { and } \sum_{\kappa} \eta^{\kappa}=+\infty \text {. }
$$

Then, either Algorithm 2 converges in a finite number of iterations to a stationary solution of (20), or every limit point of the sequence $\left\{\boldsymbol{\beta}^{\kappa}\right\}$ (at least one of such points exists) is a stationary solution of (20). Moreover, none of such points is a local minimum of the social function.

An example of sequence $\eta^{\kappa}$ satisfying conditions (27) in Theorem 3 is [9]:

$$
\eta^{\kappa}=\eta^{\kappa-1}\left(1-\epsilon \eta^{\kappa-1}\right), \kappa=1, \ldots,
$$

where $\epsilon \in(0,1)$ is a given constant.

\section{PERFormance EVAluation}

System setup. We consider a communication area with size of $200 \times 200 \mathrm{~m}^{2}$. The area is divided into 100 blocks, each having a size of $20 \times 20 \mathrm{~m}^{2}$. Then, we generate a random number of users in each block according to a Poisson distribution with different values of the density parameter $\mu$. For example, if $\mu=1$, the average number of transmitters is 0.1 in each block and is 10 in the whole communication area. Each transmitter communicates with its intended receiver that is located in an arbitrary direction with a random distance between 50 and 100 meters. The path loss factor between any two nodes is set to $\alpha=3$, and the Rayleigh fading factor is set to $\Omega=0.5$. The SINR threshold $\gamma_{\text {th }}$ to successfully decode a packet is set to a typical value of 10 .

To benchmark our algorithms, we developed a centralized solution algorithm based on the branch and bound framework to obtain the global optimum of the social problem (20). A simple "aggressive policy" is also tested to provide a performance benchmark. Based on the policy, each user uses the lowest channel fading threshold of $\beta_{n}=\beta_{n}^{\mathrm{L}}$ (which is set to $\frac{\sqrt{2}}{2} \Omega$ as discussed in Section III) and hence aggressively chooses to transmit with high probability. All results are averaged over 20 independent simulations.

Convergence performance. Convergence of Algorithm 1 is shown in Fig. 1 (a: top) for a network with average number of users set to 20. In the figure, we plot the throughput of five randomly selected users and the average fading threshold of all users against the iteration number. The average incoming packet rates $\lambda$ are randomly selected from domain of [20 60]. We observed that the distributed algorithm converges very quickly, which is usually within four-five iterations in all tests. This verifies our analysis about the convergence condition in 


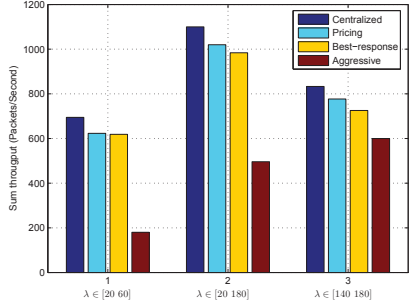

(a)

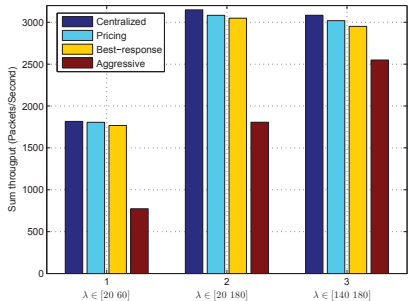

(b)
Fig. 2: Comparison of the four algorithms in high interference network with different traffic loads, (a): 15 users and (b): 50 users, two frequencies.

Section III, that is, the distributed best-response algorithm converges in networks with loosely coupled interference.

The average Rayleigh fading thresholds corresponding to the above three cases are given in Fig. (1) (a: bottom) in cases of low $\left(\lambda \in\left[\begin{array}{ll}20 & 60\end{array}\right]\right)$, moderate $\left(\lambda \in\left[\begin{array}{ll}20 & 180\end{array}\right]\right)$ and high $\left(\lambda \in\left[\begin{array}{ll}140 & 180\end{array}\right]\right)$ load traffics. The proposed distributed solution algorithms also converges very fast in all cases.

Throughput comparison. Throughput performance of the proposed distributed solution algorithm is presented in Fig. 1 (b) and Fig. 1 (c), where the average packet rate of each user is selected from [20 60] and [20 180], and the number of frequencies is set to 10 and 2, respectively. The proposed bestresponse distributed algorithm achieves good sum throughput performance. For example, Fig. 1 (b) shows that an $98.3 \%$ of the global optimum can be achieved in the case of five users and ten frequencies. It can also be seen that the aggressive policy achieves comparable (but lower) throughput only in the case of five users with ten frequencies, while it achieves a sum throughput much lower than the global optimum and the proposed best-response algorithm in all the other cases.

Finally, Fig. 1 (c) with Fig. 1 (b) show that Algorithm 1 suffers from higher price of anarchy when there is high interference among users, e.g., due to large number of users, less available frequencies or high load traffic. This performance gap can be partially filled by allowing certain degree of cooperation among users as shown in Fig. 2, where we report the performance achievable by Algorithm 2, for different traffic loads.

We can see that in case of high load traffic, Algorithm 2 achieves more than $7 \%$ of improvement in throughput with respect to Algorithm 1, whereas the gain reduces to $3.5 \%$ in case of moderate load, and it is negligible when the traffic load is low. From these results, we conclude that local cooperation (through pricing) is desirable in high interference networks (Fig. 2 (a)), whereas similar conclusion can be drawn from Fig. 2 (b), which illustrates the performance with 50 users.

\section{CONCLUSIONS}

We studied distributed queueing games in interferencelimited wireless networks. The problem of throughput maximization through distributed selection of a transmission thresh- old was formulated as a NE problem. We proposed a bestresponse based algorithm for the noncooperative case and a pricing-based algorithm for the cooperative scenario. Through comparisons to the global optimum, we showed that noncooperative best response strategies work well in general, and achieve a significant portion of (often very close to) the global optimum. In case of high interference coupling among users, the optimality gap increases, and can be partially filled by relying on cooperative strategies.

\section{REFERENCES}

[1] J. Huang, R. A. Berry, and M. L. Honig, "Distributed Interference Compensation for Wireless Networks," IEEE Journal on Selected Areas in Communications, vol. 24, no. 5, pp. 1074-1084, May 2006.

[2] K.-J. Park, C. Hou, T. Başar, and H. Kim, "Noncooperative Carrier Sense Game in Wireless Networks," IEEE Trans. on Wireless Communications, vol. 8, no. 10, pp. 5280-5289, Oct. 2009.

[3] Y. Sarikaya, T. Alpcan, and O. Ercetin, "Dynamic Pricing and Queue Stability in Wireless Random Access Games," accepted by IEEE Journal of Selected Topics in Signal Processing.

[4] L. Le, E. Modiano, and N. B. Shroff, "Longest-Queue-First Scheduling Under SINR Interference Model," in Proc. ACM International Symposium on Mobile Ad Hoc Networking and Computing (MobiHoc), Chicago, Illinois, USA, Sept. 2010.

[5] Y. Xi and E. M. Yeh, "Throughput Optimal Distributed Power Control of Stochastic Wireless Networks," IEEE/ACM Transactions on Networking, vol. 18, no. 4, pp. 1054-1066, Aug. 2010.

[6] Z. Guan, T. Melodia, D. Yuan, and D. Pados, "Distributed Spectrum Management and Relay Selection in Interference-Limited Cooperative Wireless Networks," in Proc. ACM International Conference on Mobile Computing and Networking (MobiCom), Las Vegas, USA, Sept. 2011.

[7] D. Yang, X. Fang, and G. Xue, "Truthful Auction for Cooperative Communications," in Proc. ACM International Symposium on Mobile Ad Hoc Networking and Computing (MobiHoc), Paris, France, May 2011.

[8] M. Hanawal, E. Altman, and F. Baccelli, "Stochastic Geometry based Medium Access Games," in Proc. IEEE International Conference on Computer Communications (INFOCOM), Orlando, FL, March 25-30, 2012.

[9] G. Scutari, F. Facchinei, P. Song, D. Palomar, and J.-S. Pang, "Decomposition by Partial Linearization: Parallel Optimization of Multi-Agent Systems," IEEE Trans. on Signal Processing, (submitted Jan. 2013). Available at http://arxiv.org/pdf/1302.0756v1.pdf.

[10] G. Scutari, D. P. Palomar, F. Facchinei, and J.-S. Pang, "Distributed Dynamic Pricing for MIMO Interfering Multiuser Systems: A Unified Approach," in Proc. of International Conference on NETwork Games, COntrol and OPtimization (NetGCooP 2011), October 12-14, 2011, Paris, France.

[11] T. S. Rappaport, Wireless Communications. USA: Prentice Hall PTR, 1996.

[12] Z. Guan, T. Melodia, and G. Scutari, "To Transmit or Not To Transmit? Distributed Queueing Games in Interferencelimited Wireless Networks," Technical Report, available at: http://202.194.20.8/guan/tec_rpts/TR_QueueGame.pdf.

[13] F. Baccelli and B. Blaszczyszyn, Stochastic Geometry and Wireless Networks, Part I: Theory. USA: Now Publishers Inc, 2009.

[14] J. B. Rosen, "Existence and Uniqueness of Equilibrium Points for nperson Games," Econometrica, vol. 33, no. 3, pp. 520-534, Jul. 1965.

[15] G. Scutari, D. P. Palomar, F. Facchinei, and J.-S. Pang, "Monotone Games for Cognitive Radio Systems," in Distributed Decision-Making and Control. Eds. Anders Rantzer and Rolf Johansson, Lecture Notes in Control and Information Sciences Series, Springer Verlag, 2011.

[16] G. Scutari, F. Facchinei, J.-S. Pang, and D. Palomar, "Real and Complex Monotone Games," IEEE Trans. on Information Theory, (submitted November 2012). Available at http://arxiv.org/abs/1212.6235.

[17] D. P. Bertsekas and J. N. Tsitsiklis, Parallel and Distributed Computation: Numerical Methods. USA: Athena Scientific, 1997. 